\title{
Preparation and Applications of Super-hydrophobic materials
}

\author{
Xiaohua Zhang and Shuqiong $\mathrm{Xu}^{\text {a }}$ \\ School of Mechanical and Vehicle Engineering, Lin Yi University, Shandong, China
}

\begin{abstract}
In this paper, the formation principle of hydrophobic phenomenon, the preparation and applications of hydrophobic materials are introduced. The super-hydrophobic material is a novel material with wide application and prospect. The application of status of super-hydrophobic materials in industrial and agricultural production, medical and health fields and people's daily life, in recent years, which was studied, and the prospect of development was systematically summarized.
\end{abstract}

\section{Introduction}

Super-hydrophobic material is a kind of material with special surface properties, which has the characteristics of self-cleaning, preventing dust, preventing rain and fog adhesion, snow adhesion, preventing corrosion, and so on. Therefore, super-hydrophobic material has received wide attention in the fields of scientific research, production and life.

\section{The principle of Super-hydrophobic phenomenon}

\subsection{Contact angle and Young equation}

If a drop of water is dropped onto the surface of a solid and the surface of the solid is uniform and smooth, the angle between the tangent of the water-gas interface and the tangent of the water-solid interface is called the contact angle, generally denoted by $\theta$, which is a characteristic of the mutual wettability of the solid-liquid surface, and the smaller the contact angle, the better the wettability of water to the solid. According to the size of the contact angle, there are three cases (as shown in Fig. 1): When $0<\theta<90^{\circ}$, it is hydrophilic. As shown in fig. ( a ), when $\theta=0$, it is called a complete wetting phenomenon; When $\theta=90^{\circ}$, called hydrophobic phenomenon, as shown in figure ( $\mathrm{b}$ ); when $\theta>150^{\circ}$, called super hydrophobic phenomenon, as shown in figure ( c ), It is called super hydrophobic material that can produce super hydrophobic phenomenon. Therefore, $\theta=90^{\circ}$ is a boundary for judging whether hydrophobic or not.

*Corresponding author: ${ }^{\text {a }}$ xushuqiong11@ sina.com

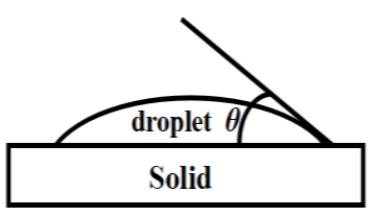

(a) Hydrophilic $\theta<90^{\circ}$

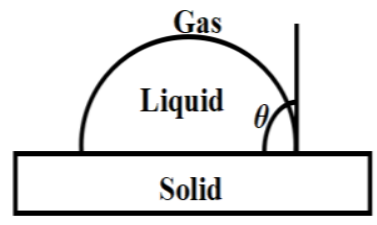

(b) Hydrophilic $\theta=90^{\circ}$

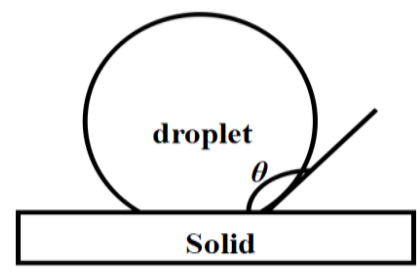

(c) Super-hydrophobic $\theta>150^{\circ}$

Figure 1. Three cases of different liquid-solid contact angles

The contact angle is the result of the balance of surface tension between solid, liquid and gas. The balance of liquid on the solid surface makes the total energy of the system tend to be minimum, so the liquid is in a stable equilibrium state on the solid surface [1] A functional relationship between the contact angle, on a three-phase line, and the surface tension of a droplet on a smooth and uniform solid surface is the Young equation:

$$
\cos \theta=\frac{\gamma_{\mathrm{sg}-} \gamma_{\mathrm{sl}}}{\gamma_{\mathrm{lg}}}
$$

That about $\gamma \mathrm{sg}, \gamma \mathrm{sl}, \gamma \mathrm{lg}$ belongs to the surface tension of solid-gas interface, solid-liquid interface and liquid-gas interface, respectively. The contact angle is the intrinsic contact angle of the material at this time. It is the basis of measuring the wettability of hydrophobic materials. Fur midge studied the contact angle hysteresis was more than 50 years ago, and the contact angle was 
related to the viscosity of the solid surface. The larger contact angle hysteresis, the stronger viscosity, that is, the more difficult the droplet is, which flowed on the solid surface; When the contact angle hysteresis is approximately equal to zero degrees, the solid surface is substantially free of viscosity, droplets tend to flow on the solid surface for the material surface is hydrophobic.

\subsection{Rolling angle}

The contact angle is one of the bases to judge the wettability of super-hydrophobic materials, and the rolling angle should be considered if the hydrophobic effect of super-hydrophobic materials is to be judged dynamically. The rolling angle at which the liquid drops onto the solid surface refers to the critical angle $\alpha$, at which the solid surface tilts, when the liquid drops on the solid surface starts to move under the influence of gravity (as shown in Fig. 2).

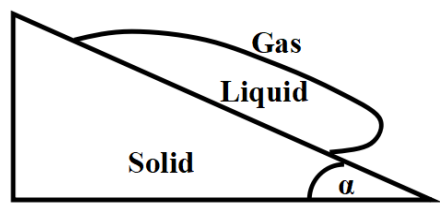

Figure 2. Rolling angle diagram of solid surface

In practice, there is a contact angle lag on the surface of the solid, at which we can point capillary forces against gravity, and the forces after equilibrium leave droplets on the surface of the solid [2].

From the above analysis, we can draw conclusions, about which the super-hydrophobic surface has a large static contact angle and a minimum contact angle hysteresis. The surface microstructure of a solid can affect the static contact angle, so that air can remain between interfaces of liquid and solid, that is, the liquid is not easy to penetrate into the rough structure. The smaller the rolling angle is, the better the hydrophobicity of the solid surface could be.

\section{The preparation of super-hydrophobic materials}

\subsection{Super- hydrophobic material with metal base}

The metal base of super hydrophobic material is made of metal as the matrix, and then the microstructure of a certain roughness is prepared on its surface and the treatment of low free energy can be carried out to achieve the effect of super hydrophobic water. Such as surface coating and other low free energy of adsorption material, for this kind of hydrophobic material use metal matrix, so, it has the certain metal properties, such as thermally conductive, anti-aging properties, therefore, which often be used in heat dissipation, pipelines, ships, etc. [3].

\subsection{Preparation of super hydrophobic surface by template method}

At present, the most commonly method of preparing super hydrophobic materials in China is the template one, which includes the soft template method and the hard template method. Soft template method is the use of poly dimethylsiloxane (PDMS) elastomer material such as soft seal or soft template, through polymer melding, printing, and preparation of super hydrophobic surface micro structure; hard template method is the use of metal substrate surface, so, as to obtain the template pressing rough surface and micro structure. Scholar named Zheng of China; he made use of $\mathrm{CaCO}_{3}$ particles as templates to prepare the on the super hydrophobic surface of polyethylene [4]. His method is that the particle size of 8 m $\mathrm{CaCO}_{3}$ particles and water $10 \%$ suspension, which will be evenly coated on the glass substrate, drying to obtain the $\mathrm{CaCO}_{3}$ particle template. Uniformly covered with a layer of linear low density of polyethylene particles in the $\mathrm{CaCO}_{3}$ template particles, the heating oven and then be placed 200 DEGC, a glass plate covered with a piece of preheating in its melting, and put them to the room temperature and then remove the insulation. Next, remove $\mathrm{CaCO}_{3}$ particles, rinse the micro melded film with water, and then immerse it in $20 \%$ concentration of hydrochloric acid for 10 minutes. At last, after drying with nitrogen, super hydrophobic surface samples are obtained.

\subsection{Fluorinated compound modification}

Fluorine compounds with low surface energy, Yabu and Shimomura fluoride glass is fixed on a movable platform, the copolymers containing fluorine casting on the glass, the wet film thickness control in less than $100 \mathrm{~m}$, adjusting the air relative humidity, temperature and the speed of the moving glass, containing fluorine honeycomb structure films with different pore size, super hydrophobic surface coating [4-5], in this sense, contact angle of 160 degrees.

\subsection{Super hydrophobic materials were prepared by phase separation}

Phase separation is the existing, join another substance in the mixed solution, the mixed solution to form a new phase, a new phase in the mixed solution is insoluble, and reuse methods of heat treatment, which will be new separation and eventually made hydrophobic material. Phase separation method mainly includes solvent-nonsolvent and emulsion. Ji Haiyan [6], such as the simple solvent-insoluble agent method, they dissolved in xylene solution of polypropylene (PP) of grain of adding an amount of acetone, and then USES the Liu Yanfa will add the PP acetone solution coated on the glass substrate, after processing on the glass surface to form a layer of white PP film. 


\subsection{Super hydrophobic materials are prepared by layers of self-assembly}

Layers of self-assembly method depends on the electrostatic interaction, make the electrically charged particles, such as layers and layers of adsorbed to the surface of a material, this method can be realized at the molecular level control of film thickness and surface chemical composition. Lin and others applied with different particle size of silica particles, as well as to the $\mathrm{pH}$ sensitive poly ally amine (PAH) acrylic acid (PAA) polyelectrolyte with a series of anion and action solution, on the glass substrate using electrostatic super hydrophilic and hydrophobic conversion[7].

\subsection{Super hydrophobic materials were prepared by sol-gel method}

Sol-gel mixture is used in accordance with the requirements, for a certain proportion of the organic or inorganic salt solution, and then adds to the shape of the nucleus, the gelation material, by controlling the gelation process and gel with spherical particles, and then after a certain temperature claiming decomposition is required for the method of phase. Breathe bay Jabs, etc. [8]. It will be treated with hydrophobic $\mathrm{SiO} 2$ nanoparticles dispersed in sol-gel matrix, and it will blend sol-SiO2 mixture evenly on cleaning, dry glass or aluminium. By changing the $\mathrm{SiO} 2$ nanoparticles in the mixture proportion and sol type, the contact angle of $166^{\circ}$, rolling angle is less than $2^{\circ}$. By which sol gel technology constructs the rough $\mathrm{TiO} 2$ in the surface of filter paper, the modified low surface energy of silage is octal three oxygen radicals after the static contact angle with water filter reaching more than $150^{\circ}$, while rolling Angle is less than $5^{\circ}$.

\section{Application of super-hydrophobic material}

\subsection{Super hydrophobic material in antifouling, water-resistant, self-cleaning application}

Super-hydrophobic materials are gradually applied to the inner and outer walls, glass surfaces and the surfaces of metal frames of buildings due to their unique hydrophobicity and self-cleaning functions, so as to achieve the effects of rain, snow, stain resistance and the others. However, at present, the products of superhydrophobic materials in building anti-pollution are mainly surface coating and protective liquid, for example, Nano super-hydrophobic self-cleaning glass coating prepared by Nakasone technology, using Nano synthesis technology. The coating has advantages of colourless, transparent, non-toxic, pollution-free and high adhesion, and has advantages of self-cleaning, anti-icing, antioxidation. Super-hydrophobic materials are also favoured by many textile industries because of their excellent anti-fouling and self-cleaning effect. Various micro-Nano structure fibres with super-hydrophobic properties can be prepared by electro spinning or surface treatment [13].

\subsection{Application of super-hydrophobic surface materials in resistance}

In our lives, pipeline transportation has become the main mode of natural gas transmission on land. However, because natural gas contains corrosive substances such as hydrogen sulphide, carbon dioxide and so on, pipeline is prone to electrochemical corrosion, erosion and other phenomena. In view of the above problems, many scholars have done a lot of research work in resistance and so on. For example, that super hydrophobic film surface lay is prepared on the surface of the aluminium and the alloy is to obviously improve the anticorrosion capability, the carbon Nano tube is bon on the surface of the aluminium plate to form a structural surface, then ,the composite surface is modified by poly to form a super-hydrophobic pet film [9].

\subsection{Application of super hydrophobic materials in the control of microfluidics}

Super hydrophobic materials are widely used in microfluidic control, which is due to their nonwetting property and low surface viscosity. Such as medically controlled droplet movement and flow, for using at the needle tip site of a micro injector to reduce attachment of expensive drugs, therefore, by reducing waste of medical supplies and contamination of the needle tip [10]. In addition, such techniques can also be applied to the field of spraying, such as making spray nozzles of super hydrophobic materials, so that sprayed droplets are more uniform and atomization effect is better [11-12].

\section{Analysis of developing super hydrophobic material}

Super-hydrophobic materials are more and more popular because of their good properties, but the preparation technology is not perfect, the manufacturing cost is high, so it cannot be widely used. At present, superhydrophobic materials are in the initial stage of continuous development, the research cost of this material is mainly production costs.

High costs are often limited to laboratory and research values due to the large number of precision instruments and large quantities of chemicals required for manufacturing. Only under the condition, large-scale production of economies of scale can make manufacturers to be more profitable, when putting into production, which need strong capital strength of large manufacturers, so that their production is limited by small manufacturers [14]. Finally, in order to realize the excellent quality and performance of super hydrophobic materials, technical personnel with relevant professional knowledge and theoretical basis are needed, which also 
increases the cost. In general, the development and application of hydrophobic materials are facing many difficulties. Firstly, the material cost is high, the low surface energy substances with hydrophobic characteristics are mostly fluorine containing or silage polymers at present, so that the batch manufacturing cost is relatively high, and the high conductive fillers ( such as Nano au, age, in and the like ) become higher prices, so, the application of raw materials is limited. Secondly, the manufacturing equipment of the material has higher requirements, harsh conditions and long cycle, which is not suitable for large-area rapid preparation, and it is still immature in industrial application. Finally, the hydrophobic property does not have good durability, and is easily polluted by oily substances or damaged surface of adhesion dust [15].

\section{Conclusions}

In recent years, more and more attention has been paid to super-hydrophobic materials. Polymer based hydrophobic materials are widely used for their low cost and simple preparation. At present, a lot of in-depth research has been carried out and it will be the most important hydrophobic materials in the future. The metal-based hydrophobic material retains the excellent properties of the metal to be a great extent, and it can meet the requirements of good conductive heat conduction, high strength and the like. However, there are lots of studies on the functionalization of super hydrophobic materials, such as directional hydrophobic, super hydrophobic, and super hydrophobic reversible switching material, with the convenient preparation process will also be an important direction for future study and research [16].

\section{References}

1. Jingou Ji, Yang Bin, Xia Zhining, et al. preparation of super-hydrophobic self-cleaning coatings of nano-composite fluorine modified acrylic resin $[\mathrm{J}]$. Journal of materials research, 2011, 25, (4): 422 426

2. Jiajun Zhao. Experimental study and numerical simulation of slip flow in super hydrophobic surface micro channels. Dalian university of technology, 2006

3. Q.Zhou,sand blastinganodic oxidation fluorination treatment to construct super hydrophobic surface of aluminum alloy. Chemical journal of Chinese universities, 2010

4. Liu Ying, Jiang Yijian. Rapid preparation of super hydrophobic polyvinylidene fluoride by exciter laser [J]. China laser, 2011

5. Haiyan Ji, Chen Gang, Hu Jie.Preparation and Properties of Biomimetic Super hydrophobic Polypropy LeneFilms. Polymer Materials Science and Engineering, 2011
6. LIN,TSAI $\mathrm{P} \quad \mathrm{S}$, et al. Fabrication and characterization of transparent super hydrophilic super drophobicsilica nanoparticulate thin films. Thin Solid Films, 2011

7. Feng Jie, Lin Yunfei, Huang Mingda, et al. Preparation of super-hydrophobic polyolefin surface by hotpressing micromolding based on metal template. Journal of chemical engineering of Chinese universities, 2011, 25 (4)

8. Zheng Jiefeng, Zhong Mingqiang. Preparation of super-hydrophobic surface by $\mathrm{CaCO} 3$ particle template method. Acta polymer silica, 2010, 10: 1186-1192

9. Zhao Yonggang, Yang Xiaomin, Zhong Huajun, et al. application of enhanced PDMS in preparation of biomimetic super-hydrophobic materials. Journal of east China Jiao Tong University, 2009

10. Feng Jie, $\mathrm{CaCO}_{3}$ particle template preparation of polymer super hydrophilichydrophobic surface J. Act polymeric Sonica, 2010

11. Yabu H, SHIMOMURAM.Single-step fabrication of transparent super hydrophobic porous polymer films .Chemistry of Materials, 2005

12. Cassie A B D, Baxter S. Wettability of Porous Surfaces.Transitions of the Faraday Society, 1944, 40:546-551

13. Basu,Harip the effect of microstructure and surfaceRoughnessontheWettabilityofSuperhydropho bicSolgelNanocompositeCoatings.2010.SogelSciTec hnol $278-286$

14. Nano rod arrays Based on Self removing Metalmonomeric Template. Journal of Colloid and Interface Science, 2012

15. Sol-gel Sic Tech no SHIRGHOLAMIM A, KHALIL ABADMS, KHAJAVIR, et al. Fabrication of super hydrophobic polymaths ilsesquioxane nanostructures on cotton textiles by absolution immersion process . Journal of Colloid \&Interface Science, 2011,

16. Zhong Wenbin, Li Yuntao, Wang Yujie, etal. Super hydrophobic Polyaniline Hollow Bars:Nano rod arrays Based on Self removing Metal-monomeric Template.Journal of Colloid and Interface Science, 2012 